\title{
Analgesia preventiva con pregabalina en pacientes postoperados de colecistectomía laparoscópica
}

\author{
Celina Trujillo-Esteves ${ }^{*}$, Nadia Helena Mayoral-Flores ${ }^{1}$, Bernardo Soto-Rivera ${ }^{1}$ y \\ José Vicente Rosas-Barrientos ${ }^{2}$ \\ ${ }^{1}$ Servicio de Anestesiología; 'Jefatura de Investigación. Hospital Regional $1 .^{\circ}$ de Octubre, Instituto de Seguridad y Servicios Sociales de los \\ Trabajadores del Estado, Ciudad de México, México
}

\section{Resumen}

Antecedentes: La analgesia preventiva es la administración de un fármaco analgésico con el objetivo de atenuar el dolor postoperatorio, la hiperalgesia y alodinia. Está justificado su uso con la finalidad de ofrecer analgesia y disminuir la ansiedad a los pacientes sometidos a procedimientos laparoscópicos. Objetivo: Evaluar si la pregabalina en dosis de $1 \mathrm{mg} / \mathrm{kg}$ de peso es eficaz para analgesia preventiva en pacientes postoperados de colecistectomía laparoscópica. Métodos: Se realizó un ensayo clínico controlado ciego simple que incluyó 60 pacientes programados para colecistectomía laparoscópica divididos en 2 grupos de manera aleatoria, donde al grupo 1 se administró placebo y al grupo 2 se le administró pregabalina una dosis diaria 72 horas previas a la intervención quirúrgica. La intensidad del dolor se evaluó mediante la Escala Numérica Analógica a la hora, 2, 6,12 y 24 horas postoperatorias, así como el nivel de ansiedad prequirúrgico con la Escala de Hamilton. Resultados: Se demostró disminución del dolor en los pacientes del grupo de pregabalina desde la primera hora $(p=0.002)$, posteriormente fue más notorio el descenso del dolor en comparación con los pacientes a los que se les dio placebo, con valor estadísticamente significativo ( $p<0.001)$, lo mismo sucedió con el nivel de ansiedad evaluada con la Escala de Hamilton ( $p$ < 005). Conclusión: El uso de pregabalina para analgesia preventiva resulta ser eficaz en la ansiedad preoperatoria y el periodo posquirúrgico, y con mínimos efectos adversos, en los pacientes operados de colecistectomía laparoscópica.

PALABRAS CLAVE: Analgesia preventiva. Dolor postoperatorio. Pregabalina. Colecistectomía laparoscópica.

\begin{abstract}
Background: Preventive analgesia is the administration of an analgesic drug with the aim of attenuating post-operative pain, hyperalgesia and allodynia. Its use is justified in order to offer analgesia and reduce anxiety in patients undergoing laparoscopic procedures. Objective: To evaluate if pregabalin in a dose of $1 \mathrm{mg} / \mathrm{kg}$ of weight is effective as preventive analgesia in post-operated laparoscopic cholecystectomy patients. Methods: A single-blind controlled clinical trial was conducted, which included 60 patients scheduled for laparoscopic cholecystectomy randomly divided into 2 groups, where Group 1 received placebo and Group 2 received pregabalin a daily dose $72 \mathrm{~h}$ prior to surgical intervention. The intensity of pain was assessed using the emergency nurses association scale at 2, 6, 12 and 24 post-operative $h$, as well as the level of presurgical anxiety with the Hamilton scale. Results: Pain reduction was demonstrated in patients in the pregabalin group from the $1^{\text {st }} h(p=0.002)$, later the decrease in pain was more noticeable compared to patients who were given placebo $(p<0.001)$, the same happened with the anxiety level evaluated with the Hamilton scale $(p<0.005)$. Conclusion: The use of pregabalin as preventive analgesia turns out to be effective in the post-operative period and the pre-operative anxiety with minimal adverse effects in the post-operated patients of laparoscopic cholecystectomy.
\end{abstract}

KEY WORDS: Preventive analgesia. Postoperative pain. Pregabalin. Laparoscopic cholecystectomy.

Correspondencia:

*Celina Trujillo-Esteves

E-mail: celitrujillo @ hotmail.com
Fecha de recepción: 06-03-2019

Fecha de aceptación: 19-03-2019

DOI: 10.24875/GMM.19005136
Gac Med Mex. 2019;155(Suppl 1):S22-S26

Disponible en PubMed www.gacetamedicademexico.com 


\section{Introducción}

La analgesia preventiva consiste en la administración de fármacos para conseguir un estado analgésico previo al trauma quirúrgico que disminuya la respuesta sensitiva periférica y central al dolor, con la cual se intenta interrumpir el círculo inflamación-dolor-hiperalgesia y aumento del estímulo doloroso'. La pregabalina es un neuromodulador utilizado en el tratamiento del dolor neuropático y varios trabajos indican que se puede administrar en la prevención del dolor postoperatorio ${ }^{2}$. El control del dolor postoperatorio en pacientes sometidos a colecistectomía laparoscópica es no solo un reto para el anestesiólogo por el tipo de dolor que presentan los pacientes, sino un compromiso con sus pacientes ${ }^{3,4}$. Al considerar al dolor postoperatorio resultado de la manipulación quirúrgica, de intensidad moderada a severa, se requiere del uso de combinaciones entre antinflamatorios no esteroideos, opiáceos y neuromoduladores, y está justificado con la finalidad de ofrecer analgesia adecuada y disminuir la ansiedad a los pacientes sometidos a colecistectomía laparoscópica ${ }^{5}$. El objetivo del presente estudio es evaluar si la pregabalina es eficaz usada como analgesia preventiva en el dolor posquirúrgico en pacientes sometidos a colecistectomía laparoscópica.

\section{Método}

Se realizó un ensayo clínico controlado, ciego, de asignación aleatoria por bloques balanceados, en una muestra de 60 pacientes. Por cálculo de tamaño muestral por estimación de proporciones se requerían 26 pacientes por grupo y se ajustó a 30 pacientes por posibles pérdidas. Se incluyeron pacientes de sexo indistinto, entre 18 y 60 años, con clasificación de estado físico American Society of Anesthesiologists (ASA) 1 y 2, seleccionados entre la población quirúrgica programada para colecistectomía laparoscópica, previo otorgamiento del consentimiento informado. El estudio se llevó a cabo bajo los principios de la bioética y fue aprobado previamente por los Comités de Investigación y Ética en el Hospital Regional $1 .^{\circ}$ de Octubre del Instituto de Seguridad y Servicios Sociales de los Trabajadores del Estado con número de registro institucional RPI 211.2012.

Durante la valoración preanestésica, si el paciente cumplía los criterios de inclusión se le invitaba a participar en el estudio y se realizaba el proceso de consentimiento informado. Se distribuyó a los pacientes en dos grupos; la forma de aleatorización fue por bloques utilizando una tabla de números aleatorios.

Ambos grupos recibieron cápsulas de características físicas similares, con contenido ajustado cercano al peso del paciente, de tal forma que el grupo tratamiento recibió $1 \mathrm{mg} / \mathrm{kg}$ peso de pregabalina y el grupo placebo cápsulas con el excipiente. Se les indicó tomar una cápsula cada 24 horas durante los tres días previos a la cirugía.

Previo al procedimiento anestésico se aplicó la Escala de Hamilton-Hunt para medir el nivel de ansiedad preoperatorio, también se realizó el registro de algún evento adverso referido por los pacientes buscados intencionalmente, como mareo, somnolencia, visión borrosa o edema en miembros pélvicos.

El procedimiento anestésico se trató que fuera similar para todos los pacientes, si así lo permitía su estado físico y sus antecedentes, de tal forma que la inducción anestésica fue con propofol $2 \mathrm{mg} / \mathrm{kg}$, la narcosis basal con fentanilo $3 \mathrm{mcg} / \mathrm{kg}$ y el relajante neuromuscular utilizado fue cisatracurio a dosis de100 mcg/kg; el mantenimiento anestésico se llevó a cabo con sevoflorano a dosis de 1-1.2 Concentración Alveolar Mínima (CAM), y finalmente la dosis del analgésico opioide fentanilo fue de $5 \mathrm{mcg} / \mathrm{kg} / \mathrm{hora}$. Se realizó el registro de los signos vitales, como frecuencia cardíaca, presión arterial no invasiva, $\mathrm{CO}_{2}$ espirado cada 5 minutos. Una vez concluido el procedimiento quirúrgico los pacientes fueron trasladados a la unidad de recuperación postanestésica en donde se realizó la evaluación de la intensidad del dolor.

La primera evaluación del dolor con la escala numérica analógica (ENA) (0 ausencia del dolor-10 dolor severo) en todos los pacientes se llevó a cabo al llegar a recuperación, a los 60 minutos, 2, 6,12 y 24 horas postoperatorias. Se registraron también signos vitales como la presión arterial no invasiva, la frecuencia cardíaca y la frecuencia respiratoria.

El control del dolor postoperatorio se realizó con la administración de ketorolaco $1 \mathrm{mg} / \mathrm{kg}$ de peso y tramadol $1 \mathrm{mg} / \mathrm{kg}$ intravenoso cada 8 horas y se llevó registro del número de rescates analgésicos.

Nuestra variable independiente fue el pretratamiento con pregabalina y la variable dependiente el dolor posquirúrgico.

\section{Análisis estadístico}

Se utilizaron medidas de frecuencia y de tendencia central. Para la estadística inferencial, en el caso de variables cuantitativas se utilizó la t de Student y la 
Tabla 1. Características basales de los pacientes de acuerdo con grupo de estudio

\begin{tabular}{|l|c|c|c|}
\hline Variable & $\begin{array}{c}\text { Grupo } \\
\text { pregabalina }\end{array}$ & $\begin{array}{c}\text { Grupo } \\
\text { placebo }\end{array}$ & p* \\
\hline Edad, años & $43.3 \pm 12.9$ & $46.9 \pm 8.5$ & 0.198 \\
\hline $\begin{array}{c}\text { Sexo, } \mathrm{n}(\%) \\
\text { Masculino } \\
\text { Femenino }\end{array}$ & $2(7)$ & $7(23)$ & 0.15 \\
\hline Peso (kg) & $28(93)$ & $23(77)$ & \\
\hline TAS (mmHg) & $71.7 \pm 14.6$ & $73.4 \pm 15.6$ & 0.665 \\
\hline TAD (mmHg) & $130.5 \pm 14.0$ & $128.2 \pm 14.8$ & 0.539 \\
\hline FC (Ipm) & $79.8 \pm 9.4$ & $78.0 \pm 10.5$ & 0.502 \\
\hline FR (Ipm) & $81.2 \pm 13.4$ & $81.8 \pm 8.7$ & 0.838 \\
\hline Hamilton 72 h previas & $13.7 \pm 2.2$ & $16.2 \pm 2.5$ & 0.442 \\
\hline $\begin{array}{l}\text { *Datos para variables cuantitativas expresados en media y desviación estándar. Prueba } \\
\text { de comparación para cuantitativas t de Student y para cualitativas ji cuadrada. }\end{array}$ \\
\hline $\begin{array}{l}\text { TAS: tensión arterial sistólica; TAD: tensión arterial diastólica; FC: frecuencia cardíaca; } \\
\text { FR: frecuencia respiratoria. }\end{array}$
\end{tabular}

prueba no paramétrica U Mann-Whitney para muestras independientes, y para las variables cualitativas, la prueba de chi cuadrada. El valor de significancia estadística se fijó en $p<0.05$.

\section{Resultados}

Se incluyeron a 60 pacientes sometidos a colecistectomía laparoscópica, los cuales fueron asignados aleatoriamente a recibir pregabalina $1 \mathrm{mg} / \mathrm{kg}$ peso vía oral cada 24 horas, durante tres días previos a la cirugía, o bien tabletas de almidón bajo el mismo esquema de administración.

Las características demográficas de la población del estudio fueron homogéneas y se muestran en la tabla 1.

Los resultados analgésicos fueron estadísticamente significativos $(p=0.001)$ en los pacientes del grupo 2 tratados con pregabalina con respecto al grupo 1 , tratado con placebo (Fig. 1).

En la evaluación del grado de ansiedad los pacientes del grupo 1 refirieron menor dificultad para iniciar y mantener el sueño $(p<0.006)$ (Fig. 2).

Respecto a los efectos adversos, el de mayor frecuencia fue el mareo, sin llegar a ser significativo estadísticamente ni obligar a suspender el tratamiento a ningún paciente (Fig. 3).

Respecto a las variables fisiológicas, la pregabalina hasta las 24 horas posquirúrgicas solo presentó variación en la frecuencia respiratoria con significancia estadística $(p=0.02)$, aun así se mantuvo con cifras fisiológicas normales $(19.2 \pm 0.57)$.

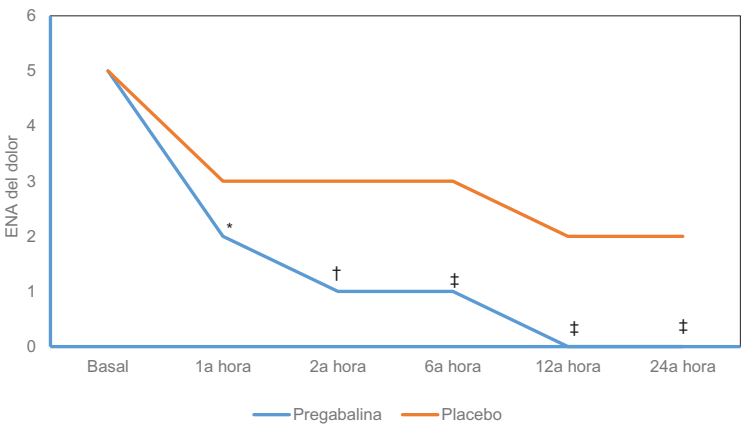

Figura 1. Comparación de de la Escala Numérica Analógica (ENA) por grupos 1a hora, 2a hora, 6a hora, 12a hora y 24 horas. ${ }^{*} p=0.002$

${ }^{\dagger} p=0.001$

${ }^{\ddagger} p<0.0001$

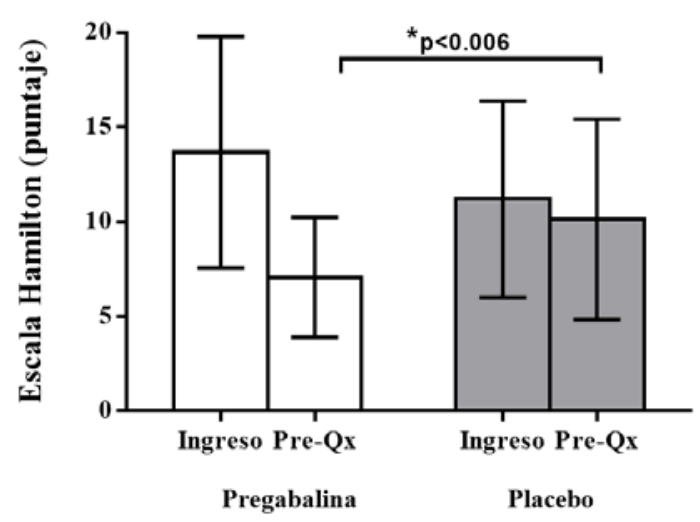

Figura 2. Comparación de calificación de ansiedad al ingreso y en el momento prequirúrgico (Pre-Qx). ${ }^{*} p<0.006$

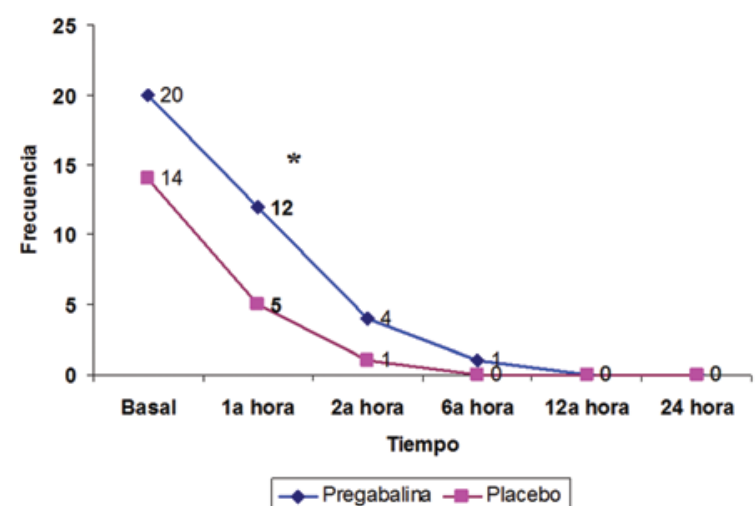

Figura 3. Frecuencia de mareo a la 1a hora, 2a hora, 6a hora, 12a hora y 24 horas. ${ }^{*} p<0.05$.

\section{Discusión}

En la colecistectomía laparoscópica el dolor tiene diferente origen y tiempo de instalación y tiene preferencia el dolor visceral y parietal en las primeras 8 
horas. En las subsiguientes 24 a 48 horas el dolor se instala en el hombro derecho principalmente y puede tener características de dolor neuropático agudo. Este dolor es ampliamente reconocido como causante de dolor poscolecistectomía laparoscópica y uno de los mecanismos responsables es la irritación del nervio frénico ${ }^{3,4}$.

En la actualidad el dolor de tipo neuropático es de difícil control y no responde de manera adecuada a los analgésicos convencionales como los antiinflamatorios no esteroideos y los analgésicos opioides, además de que con la prescripción de estos últimos siempre se tiene el riesgo de que el paciente presente náuseas y vómitos postoperatorios que pueden complicar su recuperación posquirúrgica ${ }^{6}$.

Esto da origen a la búsqueda de nuevas alternativas de tratamiento que permitan prevenir el dolor y favorezcan la disminución de las dosis de los analgésicos opioides.

La pregabalina se ha empleado para la analgesia preventiva, aunque hasta la fecha no hay consenso en relación con cuantos días de tratamiento y a qué dosis debe prescribirse para ser eficaz en el control de este síntoma y presentar un mínimo de efectos adversos?

Dworkin y Freynhagen ${ }^{8,9}$ refieren que la dosis con la que se debe iniciar la pregabalina para tener una buena analgesia posquirúrgica es de $150 \mathrm{mg} / \mathrm{día}$, administrándose $75 \mathrm{mg}$ cada 12 horas, pauta con la que ellos obtuvieron una disminución del dolor posquirúrgico de moderada a completa.

En nuestro estudio decidimos utilizar un promedio de 3 días de prescripción previos a la cirugía, puesto que encontramos diferentes estudios que la iniciaban desde una semana hasta una hora antes del procedimiento quirúrgico y también utilizamos la dosis mínima requerida de $1 \mathrm{mg} / \mathrm{kg}$ de peso cada 24 horas para buscar el efecto analgésico en el postoperatorio y determinar si a esta dosis aún existía la presencia de los efectos adversos más frecuentemente reportados con el uso de la pregabalina como son mareo, somnolencia, visión borrosa y edema perimaleolar.

Esto a diferencia de otros estudios, donde utilizaron dosis de hasta $300 \mathrm{mg}$ día, pero que se enfocan más en el efecto analgésico y en la disminución del consumo de analgésicos opioides en el periodo postoperatorio, y no buscan intencionadamente los efectos adversos que en ocasiones hacen que los pacientes tengan poco apego o incluso suspendan el tratamiento, o únicamente buscan ciertos efectos adversos como ataxia o confusión ${ }^{6,10}$.
Aun así, en relación con los efectos adversos algunos autores refieren que solo el $10 \%$ de sus pacientes los presentaron y que en $15 \%$ de estos los condicionó a suspender el medicamento. Otros autores afirman que dosis flexibles de pregabalina no disminuyen la incidencia de efectos adversos ${ }^{5,8,11}$.

En nuestro estudio aceptamos la hipótesis de que la dosis mínima de pregabalina $1 \mathrm{mg} / \mathrm{kg} / \mathrm{día}$ es efectiva como analgesia preventiva, los efectos adversos presentados fueron mínimos y no requirieron suspender el medicamento².

Trabajos previos en sujetos con dolor de similares características también comprobaron que con una sola dosis de pregabalina de 75-150 mg/día disminuía la intensidad del dolor, mientras que en nuestro estudio la dosis fue, en promedio, $75 \mathrm{mg} /$ día y se aplicó 3 días previos a la cirugía buscando obtener también un efecto ansiolítico, encontrando resultados similares en ambos estudios ${ }^{12,13}$.

Por otra parte, respecto al síntoma de ansiedad Argawal, et al. refieren que la depresión y la ansiedad son factores contribuyentes importantes para la aparición del dolor. Sharon menciona que hay múltiples factores que contribuyen al dolor, como la personalidad, la experiencia anterior de dolor y hasta factores genéticos ${ }^{8}$.

Se menciona en varios estudios que la pregabalina es un tratamiento eficaz en pacientes con trastornos de ansiedad generalizada moderada a grave. Nosotros evaluamos la intensidad de la ansiedad prequirúrgica con la Escala de Hamilton-Hunt, aunque no encontramos una disminución significativa, ya que nuestros pacientes en su mayoría solo presentaban un grado de ansiedad leve, lo cual puede estar en relación con el diagnóstico prequirúrgico y a que la cirugía laparoscópica no se considera un procedimiento de alto riesgo, lo que no ocasiona mucha ansiedad en los pacientes.

Finalmente, consideramos que para entender la variación de los resultados entre los diferentes autores y nuestro estudio hay que considerar que podría depender de dos factores, principalmente:

- Además de ser aprobado su empleo como anticonvulsivantes, la capacidad antinociceptiva de los gabapentinoides se reconoció en la década de 1990 y posteriormente se aceptó su uso en el tratamiento del dolor crónico neuropático de origen periférico, en trastornos de ansiedad generalizada y como parte de la analgesia multimodal en el perioperatorio ${ }^{14}$. 
- La analgesia preventiva aún no es una práctica común para el tratamiento del dolor posquirúrgico, la controversia en las evidencias sobre su eficacia conllevan que sea una opción aún no viable para la clínica habitual y más difícilmente si se considera el uso de gabapentinoides.

Aunque el número de estudios publicados que evalúan el papel de la pregabalina en el escenario del dolor postoperatorio es muy limitado, los resultados encontrados son prometedores no solo en el alivio del dolor en este periodo sino en la disminución de la ansiedad en cualquier tipo de cirugía. Existe una coincidencia en cuanto a que pregabalina disminuye significativamente el requerimiento de analgésicos opioides durante los periodos transanestésicos y principalmente postanestésicos, así como que también disminuye el consumo de antiinflamatorios no esteroideos y disminuye el dolor posquirúrgico, con pocos efectos adversos ${ }^{10,12,15}$.

Finalmente consideramos que al ser el dolor un síntoma subjetivo que puede ser modificado por numerosas variables, el siguiente paso en nuestra investigación es buscar el control del dolor comparando diferentes dosis y a su vez con la disminución del requerimiento de analgésicos opioides para realmente hacer objetivo el efecto analgésico de los gabapentinoides en el periodo perioperatorio.

\section{Bibliografía}

1. Sáez VP. Revisión: efecto de la analgesia preventiva en el dolor postoperatorio. Rev Esp Anestesiol Reanim. 2012;59:43-50.
2. Mishra R, Tripathi M, Chandola HJA. Comparative clinical study of gabapentin and pregabalin for postoperative analgesia in laparoscopic cholecystectomy. Anesth Essays Res. 2016;10:201.

3. Gurunathan U, Rapchuk IL, King G, Barnett AG, Fraser JF. The effect of pregabalin and celecoxib on the analgesic requirements after laparoscopic cholecystectomy: a randomized controlled trial. J Anesth. 2016;30:64-71.

4. Baron R, Mayoral V, Leijon G, Binder A, Steigerwald I, Serpell MJ, et al. $5 \%$ lidocaine medicated plaster versus pregabalin in post-herpetic neuralgia and diabetic polyneuropathy: an open-label, non-inferiority two-stage RCT study. Curr Med Res Opin. 2009;25:1663-76.

5. Buvanendran A, Kroin JS, Della Valle CJ, Kari M, Moric M, Tuman K, et al. Perioperative oral pregabalin reduces chronic pain after total knee arthroplasty: a prospective, randomized, controlled trial. Anesth Analg. 2010;110:199-207.

6. Schulmeyer MCC, de la Maza J, Ovalle C, Farias C, Vives I. Analgesic effects of a single preoperative dose of pregabalin after laparoscopic sleeve gastrectomy. Obes Surg. 2010;20:1678-81.

7. Karaca O, Pınar HU, Turk E, Dogan R, Ahiskalioglu A, Solak SK. Effects of single-dose preemptive pregabalin and intravenous ibuprofen on postoperative opioid consumption and acute pain after laparoscopic cholecystectomy. Cholecystectomy J Invest Surg 2017;20:1-7.

8. Agarwal A, Gautam S, Gupta D, Agarwal S, Singh P, Singh UJ. Evaluation of a single preoperative dose of pregabalin for attenuation of postoperative pain after laparoscopic cholecystectomy. Br J Anaesth 2008; 101:700-4.

9. O'Connor AB, Dworkin RH. Treatment of neuropathic pain: an overview of recent guidelines. Am J Med 2009; 122: S22-S32.

10. Balaban F, Yağar S, Özgök A, Koç M, Güllapoğlu HJ. A randomized, placebo-controlled study of pregabalin for postoperative pain intensity after laparoscopic cholecystectomy. J Clin Anesth. 2012;24:175-8.

11. Jokela R, Ahonen J, Tallgren M, Haanpää M, Korttila KJP. A randomized controlled trial of perioperative administration of pregabalin for pain after laparoscopic hysterectomy. Br J Anaesth. 2008 ; 134:106-12.

12. Gupta $P$, Saxena A, Chaudhary LJA. Effect of pregabalin premedication on the requirement of anesthetic and analgesic drugs in laparoscopic cholecystectomy: Randomized Comparison of Two Doses. Anesth Essays Res. 2017; 11:330-333.

13. Sarakatsianou C, Theodorou E, Georgopoulou S, Stamatiou G, Tzovaras GJ. Effect of pre-emptive pregabalin on pain intensity and postoperative morphine consumption after laparoscopic cholecystectomy. Anesth Essays Res. 2017; 11:330-333.

14. Shimony N, Amit U, Minz B, Grossman R, Dany MA, Gonen L, et al. Perioperative pregabalin for reducing pain, analgesic consumption, and anxiety and enhancing sleep quality in elective neurosurgical patients: a prospective, randomized, double-blind, and controlled clinical study. J Neurosurg. $2016 ; 125: 1513-1522$

15. Esmat IM, Farag HM. Comparative study between paracetamol and two different doses of pregabalin on postoperative pain in laparoscopic cholecystectomy. Saudi J Anaesth. 2015;9:376-80. 